\title{
The early B-type eclipsing binary GT Cephei: a massive triple system?
}

\author{
Ö. Çakırlı ${ }^{\mathrm{a}, *}$ \\ ${ }^{a}$ Ege University, Science Faculty, Astronomy and Space Sciences Dept., 35100 Bornova, \\ Izmir, Turkey
}

\begin{abstract}
GT Cep is a semi-detached close binary system with an orbital period of 4.91 days, containing a massive star. I have obtained spectroscopic observations and derived radial velocities of both components. Combining the analyses of radial velocities and available photometric observations we have measured the absolute parameters of both components of GT Cep. The components are shown to be a B2 $\mathrm{V}$ primary with a mass $\mathrm{M}_{p}=10.70 \pm 0.50 \mathrm{M}_{\odot}$ and radius $\mathrm{R}_{p}=6.83 \pm 0.19 \mathrm{R}_{\odot}$ and a A0IV secondary with a mass $\mathrm{M}_{s}=2.58 \pm 0.14 \mathrm{M}_{\odot}$ and radius $\mathrm{R}_{s}=7.56 \pm 0.21 \mathrm{R}_{\odot}$. My analyses show that GT Cep is a classical Algol-type binary with a less massive secondary filling its Roche lobe. Using the UBVJHK magnitudes and the interstellar reddening of $\mathrm{E}(\mathrm{B}-\mathrm{V})=0.61 \mathrm{I}$ estimated the mean distance to the system as $854 \pm 43 \mathrm{pc}$. The O-C residuals have been analyzed as the consequence of a light-time effect superimposed on an upward parabola. My analysis indicates that the eclipsing binary revolves around a third-body with a period of about $57.5 \mathrm{yr}$ in an orbit with a radius of $40 \mathrm{AU}$. The lower limit for the mass of the third star has been estimated
\end{abstract}

\footnotetext{
*Corresponding author. Tel.: +902323111740; Fax: +902323731403

E-mail address: omur.cakirli@gmail.com
} 
to be $7 \mathrm{M}_{\odot}$ for the inclination between $70^{\circ}$ and $90^{\circ}$.

Keywords: stars: binaries: eclipsing - stars: fundamental parameters stars: binaries: spectroscopic - stars:GT Cep

\section{INTRODUCTION}

One of the most important parameters in stellar astrophysics is the mass of stars. Eclipsing binaries with well-defined multi-passband light curves and accurate radial velocities for both components provide us with definitive empirical masses, radii, effective temperatures and luminosities. In order to better understand the physics of binary systems and test the predictions of theoretical models, it is thus important to quantitatively analyse the properties of massive binary systems with well-constrained orbital parameters. In this context studies of the rare early B-type massive stars has major highlights.

The relatively bright eclipsing binary GT Cep (HD 217224, HIP113385, $\mathrm{V}=8.13, \mathrm{~B}-\mathrm{V}=0.34$ ) was discovered to be an eclipsing binary system by

Strohmeier, Knigge, \& Ott (1962), who derived an orbital period of $4.908756 \mathrm{~d}$ using the 12 times of minima obtained from photographic observations. They obtained the first photographic light curve of the system and classified it as an Algol-type binary. A first spectroscopic study was carried out by Fitzgerald (1964) who obtained the spectroscopic orbit and classified the primary component as a B3 star. Later on the photographic study made by Bondarenko \& Tokareva (1975) who revised the orbital period and obtained a light curve containing rather a deep primary minimum and a shallow secondary minimum. Bartolini. Bonifazi, \& Milano (1984) obtained UBV light 
curves and analyzed using the Wood's method.

Photometric observations of GT Cep were also obtained by the Hipparcos satellite (GT Cep being identified as HIP 113385 (ESA, 1997)) and later in the context of the Northern Sky Variability Survey (Wozniak et al., 2004). Although GT Cep has been studied on many occasions its astrophysical parameters were not firmly established. On the other hand Ibanoglu et al. (2006) divided the semi-detached Algol-type binaries (SDABs) into two groups. The orbital angular momenta of SDABs with periods $\mathrm{P}<5$ days and $\mathrm{P}>5$ days are 45 and 25 per cent smaller than those detached binaries with similar mass. The specific angular momenta of systems with $\mathrm{P}>5 \mathrm{~d}$ are larger than than those of $\mathrm{P}<5 \mathrm{~d}$ for the gainers of the same mass. The spins of the mass gaining stars point out a sharp distinction between short and long period orbit systems at an orbital period of 5 days. The orbital period of GT Cep is very close to this discriminating period.

This paper is organized as follows. I present new spectroscopic observations and radial velocities of both components of the eclipsing pair. By analysing the previously published light curves and the new radial velocities I obtain orbital parameters for the components. Combining the results of these analyses we obtain absolute physical parameters of both components. In addition, I conclude with a brief discussion of the system's evolutionary status.

\section{OBSERVATIONS}

The present study is the result of a collaboration in which two data sets were obtained at two different in the roughly same latitude observatories, 
using the two telescopes and spectrographs.

The first dataset was obtained with the REOSC Echelle spectrograph mounted on the $182 \mathrm{~cm}$ telescope at the Asiago Observatory in Italy, with exposure time ranging from 30 to 45 minutes. The instrument covers the spectral domain between 3900 and $7300 \AA$, divided into 27 orders. The average signal-to-noise ratio $(\mathrm{S} / \mathrm{N})$ and resolving power $\lambda / \Delta \lambda$ were about 120 and $\sim 50000$, respectively. Four échelle spectra of GT Cep were taken from March 17, 2009 to July 8, 2011.

12 échelle spectra of GT Cep were collected with the Turkish Faint Object Spectrograph Camera (TFOSC)1 attached to the $1.5 \mathrm{~m}$ telescope between August 22, 2011 and August 2, 2013, under good seeing conditions. Further details on the telescope and the spectrograph can be found at http://www.tug.tubitak.gov.tr. The wavelength coverage of each spectrum was 4000-9000 $\AA$ in 12 orders, with a resolving power of $\lambda / \Delta \lambda \sim 7000$ at $6563 \AA$ and an average signal-tonoise ratio $(\mathrm{S} / \mathrm{N})$ was $\sim 120$. I also obtained high $\mathrm{S} / \mathrm{N}$ spectra of the early type standard stars 1 Cas (B0.5 IV), HR 153 (B2 IV), $\tau$ Her (B5 IV), 21 Peg $(\mathrm{B} 9.5 \mathrm{~V})$ and $\alpha \operatorname{Lyr}(\mathrm{A} 0 \mathrm{~V})$ for use as templates in derivation of the radial velocities.

I applied the same reduction procedure to both datasets. The electronic bias was removed from each image and I used the 'crreject' option for cosmic ray removal. Thus, the resulting spectra were largely cleaned from the cosmic rays. The échelle spectra were extracted and wavelength calibrated by using Fe-Ar lamp source with help of the IRAF 2 ECHELle PACKAGE (Simkin,

\footnotetext{
${ }^{1}$ http://tug.tug.tubitak.gov.tr/rtt150_tfosc.php

${ }^{2}$ IRAF is distributed by the National Optical Astronomy Observatory, which is op-
} 
1974).

\section{RADIAL VELOCITIES AND ATMOSPHERIC PARAMETERS}

\subsection{Period Determination}

A total of 22 times of mid-primary minimum and one secondary of GT Cep were collected from the literature and listed in Table 1. The starting epoch and orbital period are taken from the Hipparcos and Kreiner (2004), respectively. Therefore the following ephemeris was used to determine the cycle number and $O-C(\mathrm{I})$ residuals,

$$
\operatorname{MinI}(H J D)=2448503.19+4^{d} .9087946 \times E .
$$

The O-C(I) residuals, indicating the differences between observed times of mid-eclipses and calculated ones using this ephemeris are listed in the third column of Table1. These residuals for all the times of mid-eclipses of the GTCep are plotted against the epoch numbers in the top panel of Fig. 1. The trend of the $O-C(\mathrm{I})$ residulas can be described by an upward parabolic curve superimposed on a sine-like variation. It is obvious that the change of the $O-C(\mathrm{I})$ residuals is a result of at least two separate causes. Because GT Cep is a semi-detached Algol-type binary, the system could be transferring mass from less massive component to the more massive primary leading to an upward parabolic change of orbital period, i.e., indicating that the orbital period is continuously increasing.

erated by the Association of Universities for Research in Astronomy,Inc. (AURA), under cooperative agreement with the National Science Foundation 
Recently Liao \& Qian (2010) suggested that cyclic period changes are a common phenomenon in close binary systems. Cyclic variations in the orbital periods are usually explained by magnetic activity in one or both components, by an apsidal motion, and or by the light-travel time effect around commoncenter with a third-body. Since GT Cep is composed of a B2 V and a A0 IV star that contain convective core and radiative atmosphere. This suggests that the cyclic changes in the $O-C$ residuals can not be originated from the magnetic activity cycle mechanism. We can, therefore, easily ignore the possibility of solar-like activity in both comments. Both the light and radial velocities of the system point out a circular orbit for the system. In addition, the $\mathrm{O}-\mathrm{C}(\mathrm{I})$ residual obtained for the mid-secondary eclipse seem to follow the same trend as the primary minimum. Therefore, we may rule out the apsidal motion as a possible cause of orbital period change. Therefore such a sinusoidal/cyclic change in the orbital period of GT Cep can only be explained by an orbital motion around a third-body. We analyzed the O$\mathrm{C}(\mathrm{I})$ residuals under an assumption of a combination of mass-transfer and third-body, i.e. the eclipsing pair is orbiting around a third-body. We may compute the times of light minimum with a formula as

$$
T_{\mathrm{ec}}=T_{1}+P_{1} \times E+Q \times E^{2}+\delta T .
$$

where $T_{1}$ is the starting epoch, $\mathrm{E}$ is the integer eclipse number and $\mathrm{P}_{1}$ is the orbital period of the eclipsing pair. While the third-term represents the parabolic change in the $O-C$ residuals the time delay or advance of any observed eclipse is caused by the influence of a third-body can be represented by a fourth-term. The light-time effect $\delta \mathrm{T}$ is depended up on the semi-major 
axis of the eclipsing pair around the barycenter, inclination, eccentricity and longitude of the periastron of the third-body orbit. I have used the conventional formulae given by Ibanoglu et al. (2000). A linear least squares solution was applied to the data and the coefficient of the third-term and the parameters for the third-body orbit were obtained. The coefficient of the quadratic-term, originated from the mass transfer between the components is calculated as $\mathrm{Q}=1.28 \times 10^{-9} \pm 0.22 \times 10^{-9}$ days cycle $^{-1}$. A secular period increase has been calculated as $\mathrm{dP} / \mathrm{dt}=1.90 \times 10^{-7} \mathrm{~d} \mathrm{yr}^{-1}$ which corresponds to 1.64 sec century $^{-1}$, indicating that mass transfer from less massive to the more massive star at a rate of $\mathrm{dM} / \mathrm{dt}=4.4 \times 10^{-8} \mathrm{M}_{\odot} \mathrm{yr}^{-1}$.

The parameters of the third-body orbit are listed in Table 2. My calculation suggests that the eclipsing pair revolves around a third-body, in an eccentric orbit with $\mathrm{e}=0.047$, and with a period of about $57.5 \pm 2.3$ years. The projected radius of the orbit of the eclipsing pair around the center-ofmass is about $13.80 \pm 0.52 A U$. Using these values I obtained a mass function as $0.796 \pm 0.060 \mathrm{M}_{\odot}$. The $\mathrm{O}-\mathrm{C}(\mathrm{II})$ residuals were obtained after subtracting the continuous period increase and the light-time effect, and are plotted in the bottom panel of Fig. 1.

\subsection{Radial velocity}

To derive the radial velocities, the sixteen spectra obtained for the system are cross correlated against the template spectra of standard stars 1 Cas, HR 153, $\tau$ Her, 21 Peg and $\alpha$ Lyr on an order-by-order basis using the FXCOR package in IRAF. The standard stars' spectra were synthetically broadened by convolution with the broadening function of Gray (1992). The crosscorrelation with the standard star $\tau$ Her gave the best result. I have also 


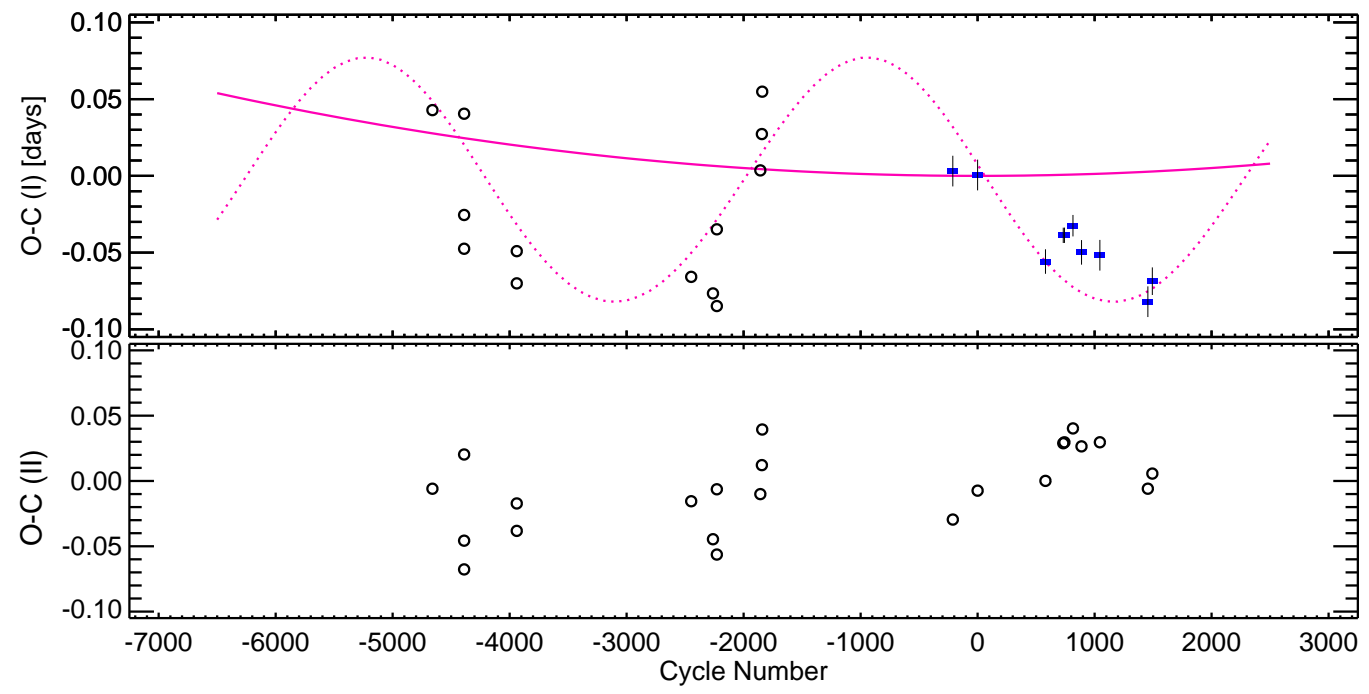

Figure 1: The $O-C(\mathrm{I})$ diagram for GT Cep obtained using the Eq.(1) and its representation with a quadratic-term (solid line) and a light-time effect (dotted line). In the bottom panel I plotted the $O-C(\mathrm{II})$ residuals, deviations from the upward parabola and a light-time fit, versus the epoch numbers. The filled lying-bar with error bars refer to photoelectric times for mid-minima and open circles refer to photographic ones. 
Table 1: Literature times of minimum light of GT Cep and the observed minus calculated $(O-C)$ values of the data obtained by Eqs.(2) and (3). References: (1) (Strohmeier 1962); (2) (Berthold 1976); (3) (Diethelm 2003); (4) (Kreiner 2004); (5) (Meyer 2006); (devil) (VSX-O-C Gateway-http://var.astro.cz/ocgate/ocgate.php); (7) (ESA 1997); (music) (Wozniak et al. 2004).

\begin{tabular}{|c|c|c|c|c|c|}
\hline $\begin{array}{l}\text { Time of minimum } \\
(\text { HJD }-2400000)\end{array}$ & $\begin{array}{c}\text { Cycle } \\
\text { number }\end{array}$ & $\begin{array}{l}\text { O-C }(\mathrm{I}) \\
(\text { days })\end{array}$ & $\begin{array}{r}\text { O-C }(\mathrm{II}) \\
(\text { days })\end{array}$ & Type & Reference \\
\hline 25628.250 & -4660 & 0.043 & -0.006 & $\mathrm{pg}$ & 1 \\
\hline 26958.443 & -4389 & -0.048 & -0.068 & pg & 1 \\
\hline 26958.465 & -4389 & -0.026 & -0.046 & $\mathrm{pg}$ & 1 \\
\hline 26958.531 & -4389 & 0.040 & 0.020 & $\mathrm{pg}$ & 1 \\
\hline 29167.378 & -3939 & -0.070 & -0.038 & pg & 1 \\
\hline 29167.399 & -3939 & -0.049 & -0.017 & $\mathrm{pg}$ & 1 \\
\hline 36486.395 & -2448 & -0.066 & -0.016 & $\mathrm{pg}$ & 2 \\
\hline 37399.420 & -2262 & -0.077 & -0.045 & $\mathrm{pg}$ & 2 \\
\hline 37561.402 & -2229 & -0.085 & -0.056 & $\mathrm{pg}$ & 2 \\
\hline 37561.452 & -2229 & -0.035 & -0.006 & $\mathrm{pg}$ & 2 \\
\hline 39387.562 & -1857 & 0.004 & -0.010 & pg & 6 \\
\hline 39451.400 & -1844 & 0.027 & 0.012 & $\mathrm{pg}$ & 6 \\
\hline 39466.154 & -1841 & 0.055 & 0.039 & pg & 6 \\
\hline 47462.5279 & -212 & 0.0024 & -0.0295 & pe & 6 \\
\hline 48503.190 & 0 & 0.000 & -0.007 & pe & 7 \\
\hline 51350.235 & 580 & -0.056 & 0.000 & pe & 8 \\
\hline 52096.389 & 732 & -0.039 & 0.029 & pe & 3 \\
\hline 52145.477 & 742 & -0.039 & 0.030 & pe & 3 \\
\hline 52503.8253 & 815 & -0.0323 & 0.0403 & pe & 6 \\
\hline 52862.150 & 888 & -0.050 & 0.027 & pe & 4 \\
\hline 53632.829 & 1045 & -0.051 & 0.030 & pe & 5 \\
\hline 55645.4049 & 1455 & -0.0812 & -0.0059 & pe & 6 \\
\hline 55834.4069 & 1493.5 & -0.0678 & 0.0057 & pe & 6 \\
\hline
\end{tabular}


Table 2: Orbital solution for the third component of

\begin{tabular}{lcc} 
GT Cep. & & \\
\hline Parameter & Value & $\sigma$ \\
\hline $\mathrm{T}_{1}$ (HJD) & 2448503.1967 & 0.0011 \\
$\mathrm{P}_{1}$ (day) & 4.908803 & 0.000003 \\
$A$ (day) & 0.080 & 0.002 \\
$e$ & 0.047 & 0.005 \\
$\omega$ & 41 & 2 \\
$T_{3}$ (HJD) & 2440956.21 & 0.17 \\
$P_{3}$ (year) & 57.5 & 2.3 \\
$a_{12} \sin i$ (AU) & 13.80 & 0.52 \\
\hline
\end{tabular}

used $\alpha$ Lyr for some spectra.

The spectra showed two distinct cross-correlation peaks in the quadratures, one for each component of the binary. Thus, both peaks are fitted independently with a Gaussian profile to measure the velocities and their errors for the individual components. If the two peaks appear blended, a double Gaussian was applied to the combined profile using de-blend function in the task. I applied the cross-correlation technique to two wavelength regions with well-defined absorption lines of the primary and secondary components. These regions (3rd and 4th orders) include the HeI $\lambda 6678$ and $\lambda 5876 \AA$ lines, dominant in early B-type stars. Here I used as weights the inverse of the variance of the radial velocity measurements in each order, as reported by FXCOR. I have been able to measure radial velocities of both components with a precision below than $10 \mathrm{~km} \mathrm{~s}^{-1}$.

The heliocentric radial velocities for the primary $\left(\mathrm{V}_{p}\right)$ and the secondary $\left(\mathrm{V}_{s}\right)$ components are listed in Table 3, along with the dates of observations and the corresponding orbital phases computed with the new ephemeris given in Eq.2. The velocities in this table have been corrected to the heliocentric 


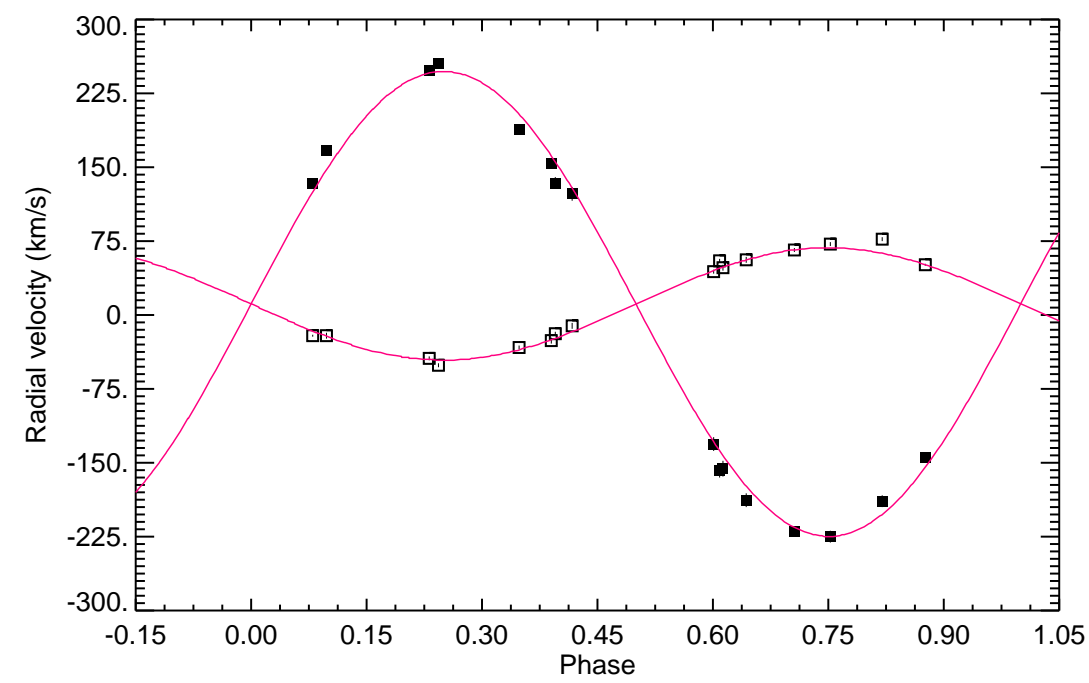

Figure 2: Radial velocities for the components of GT Cep. Symbols with error bars, generally smaller than the symbol size, show the radial velocity measurements for the components of the system (primary: open squares, secondary: filled squares).

reference system by adopting a radial velocity value for the template stars. The radial velocities are plotted against the orbital phase in Fig. 2 where the empty squares correspond to the primary and the filled squares to the secondary star. I have analyzed all the radial velocities for the initial orbital parameters using the RVSIM software program Kane, Schneider, \& Ge (2007). The best fit was obtained for a circular orbit with a mass-ratio of $q=\frac{M_{2}}{M_{1}}=0.2415 \pm 0.0094$ and a projected separation of $\operatorname{asin} i=28.42 \pm 0.43$ $\mathrm{R}_{\odot}$. The orbital solution is presented in Table 4 . The continuous lines in Fig. 2 show the computed curves. 
Table 3: Heliocentric radial velocities of GT Cep. The columns give the heliocentric Julian date, the orbital phase (according to the ephemeris in $\S 3$ ), the radial velocities of the two components with the corresponding standard deviations.

\begin{tabular}{cccccc}
\hline HJD 2400000+ & Phase & \multicolumn{2}{c}{ Star 1} & \multicolumn{2}{c}{ Star 2} \\
& & $V_{p}$ & $\sigma$ & $V_{s}$ & $\sigma$ \\
\hline 54908.5442 & 0.8760 & 51 & 1 & -145 & 2 \\
54909.5455 & 0.0800 & -21 & 1 & 133 & 2 \\
55346.5162 & 0.0979 & -21 & 3 & 167 & 4 \\
55751.5070 & 0.6010 & 44 & 2 & -131 & 7 \\
55796.4306 & 0.7526 & 72 & 2 & -225 & 6 \\
55835.4714 & 0.7059 & 66 & 2 & -220 & 4 \\
56130.5596 & 0.8200 & 77 & 2 & -189 & 6 \\
56132.5791 & 0.2314 & -44 & 2 & 248 & 4 \\
56133.4902 & 0.4171 & -11 & 3 & 123 & 7 \\
56134.6009 & 0.6433 & 56 & 3 & -188 & 7 \\
56137.5467 & 0.2434 & -51 & 2 & 255 & 2 \\
56167.5138 & 0.3482 & -33 & 2 & 188 & 2 \\
56506.4260 & 0.3900 & -26 & 3 & 154 & 5 \\
56506.4516 & 0.3952 & -19 & 2 & 134 & 6 \\
56507.4997 & 0.6087 & 55 & 2 & -158 & 7 \\
56507.5210 & 0.6131 & 48 & 3 & -156 & 8 \\
\hline & & & & &
\end{tabular}

Table 4: Orbital solution of the Algol-type binary in GT Cep.

\begin{tabular}{ccc}
\hline Parameter & \multicolumn{2}{c}{ GT Cep } \\
& Primary & Secondary \\
\hline $\mathrm{K}\left(\mathrm{km} \mathrm{s}^{-1}\right)$ & $57 \pm 2$ & $236 \pm 4$ \\
$\mathrm{~V}_{\gamma}\left(\mathrm{km} \mathrm{s}^{-1}\right)$ & \multicolumn{2}{c}{$11 \pm 1$} \\
Average $\mathrm{O}-\mathrm{C}\left(\mathrm{km} \mathrm{s}^{-1}\right)$ & 1.6 & 2.5 \\
$\mathrm{M} \sin ^{3} i\left(\mathrm{M}_{\odot}\right)$ & $10.30 \pm 0.48$ & $2.49 \pm 0.14$ \\
Mass ratio, $q$ & $0.2415 \pm 0.0094$ \\
$a \sin i\left(\mathrm{R}_{\odot}\right)$ & \multicolumn{2}{c}{$28.42 \pm 0.43$} \\
\hline
\end{tabular}




\subsection{Determination of the atmospheric parameters}

Mid-resolution optical spectroscopy permits us to derive most of the fundamental stellar parameters, such as projected rotational velocity $(V \sin i)$, spectral type $\left(\mathrm{S}_{p}\right)$, luminosity class, effective temperature $\left(\mathrm{T}_{\text {eff }}\right)$, surface gravity $(\log g)$, and metallicity $([\mathrm{Fe} / \mathrm{H}])$.

The width of the cross-correlation function (CCF, hereafter) is a good tool for the measurement of projected rotational velocity $(V \sin i)$ of a star. I use a method developed by Penny (1996) to estimate the $V$ sin $i$ of each star composing the investigated SB2 system from its CCF peak by a proper calibration based on a spectrum of a narrow-lined star with similar spectral type. For the system, the rotational velocities of the components were obtained by measuring the FWHM of the CCF peak related to each component in five high-S/N spectra acquired near the quadratures, where the spectral lines have the largest Doppler-shift. The CCFs were used for the determination of $V \sin i$ through a calibration of the full-width at half maximum (FWHM) of the CCF peak as a function of the $v \sin i$ of artificially broadened spectra of slowly rotating standard star $\left(21 \mathrm{Peg}, V \sin i=14 \mathrm{~km} \mathrm{~s}^{-1}\right.$, e.g., Rover et al. (2002)) acquired with the same setup and in the same observing night as the target systems. The limb darkening coefficient was fixed at the theoretically predicted values, 0.42 for both components van Hamme (1993). We calibrated the relationship between the CCF Gaussian width and $V \sin i$ using the Conti \& Ebbets (1977) data sample. This analysis yielded projected rotational velocities for the components of GTCep as $V_{p} \sin i=70 \mathrm{~km} \mathrm{~s}^{-1}$, and $V_{s} \sin i=70 \mathrm{~km} \mathrm{~s}^{-1}$. The mean deviations were 4 and $7 \mathrm{~km} \mathrm{~s}^{-1}$, for the primary and secondary, respectively, between the measured velocities for dif- 
ferent lines.

We also performed a spectral classification for the components of the system using COMPO2, an IDL code for the analysis of high-resolution spectra of SB2 systems (see, e.g., Cakirli et al. 2014) and adapted to the TFOSC spectra. This code searches for the best combination of two reference spectra able to reproduce the observed spectrum of the system. We give, as input parameters, the radial velocities and projected rotational velocities $v \sin i$ of the two components, which were already derived. The code then finds, for the selected spectral region, the spectral types and fractional flux contributions that better reproduce the observed spectrum, i.e. which minimize the residuals in the collection of difference (observedcomposite) spectra. For this task we used reference spectra taken from the Valdes et al. (2004) Indo U.S. Library of Coude Feed Stellar Spectra (with a a resolution of $\approx 1 \AA$ ) that are representative of stars with various metallicity type, spectral types from late-O type to early-A, and luminosity classes V, IV, and III. The atmospheric parameters of these reference stars were recently revised by Wu et al. (2011). We selected 198 reference spectra spanning the ranges of expected atmospheric parameters, which means that we have searched for the best combination of spectra among 39204 possibilities per each spectrum. The observed spectra of GT Cep in the $\lambda \lambda 6525$ - 6720 spectral region were best represented by the combination of HD 886 (B2 V) and HD 77350 (A0 IV). We have derived a spectral type of B2 main sequence star for the primary and A0 sub-giant for the secondary star of GTCep, with an uncertainty of about 0.5 spectral subclass, by adopting the spectral type and luminosity class which are more frequently encountered. The effective temperature 
Table 5: Spectral types, effective temperatures, surface gravities, and rotational velocities of each components estimated from the spectra of GT Cep.

\begin{tabular}{lcc}
\hline Parameter & \multicolumn{2}{c}{ GT Cep } \\
& Primary & Secondary \\
\hline Spectral type & B2 V & A0 IV \\
$\mathrm{T}_{\text {eff }}(\mathrm{K})$ & $22400 \pm 950$ & $10100 \pm 650$ \\
$\log g($ cgs $)$ & $3.86 \pm 0.05$ & $3.16 \pm 0.17$ \\
$V \sin i\left(\mathrm{~km} \mathrm{~s}^{-1}\right)$ & $70 \pm 4$ & $70 \pm 7$ \\
\hline
\end{tabular}

and surface gravity of the two components of each system are obtained as the weighted average of the values of the best combinations of templates adopting a weight $w_{i}=1 / \sigma_{i}^{2}$, where $\sigma_{i}$ is the average of residuals for the $i$-th combination. The standard error of the weighted mean was adopted for the atmospheric parameters. Both stars appear to have a solar metallicity, within the errors. The atmospheric parameters obtained by the code and their standard errors are reported in Table 5. The observed spectra of GT Cep at phases near to the quadratures are shown in Fig. 3 \& 4 together with the combination of two reference spectra which give the best match. The arrows in the top right panel of Fig. 4 show C II $\lambda \lambda 6578$ and 6583 lines.

\section{ANALYSES OF THE LIGHT CURVES}

The photographic BV observations were collected by Bondarenko \& Tokareva (1975). The first photometric elements of the system were calculated using the express method of Tsesesvich. Later on, the system was observed photoelectrically by Bartolini, Bonifazi, \& Milano (1984). The almost complete UBV light curves were obtained and analyzed by the methods Russell Merrill, Kitamura and Wilson - Devinney. Recent light curves of the 




Figure 3: Four spectra of GT Cep near opposing quadrature phases. The wavelength limits are 6650-6700 , which include the He I $\lambda 6678$ line. The deeper lines in each spectra refer to the primary star $(\mathrm{P})$ and the shallower lines to the secondary (S). Vertical axis is the normalized flux. 




Figure 4: Four spectra of GT Cep near opposing quadrature phases. The wavelength limits are 6500-6600 $\AA$, which include the $\mathrm{H} \alpha$ line. The deeper lines in each spectra refer to the primary star (P) and the shallowers to the secondary star (S). Vertical axis is the normalized flux. 
Table 6: Results of the analyses of the light curves for GT Cep.

\begin{tabular}{lcccc}
\hline Parameter & Bartolini et al (1984) & Bondarenko and Tokareva (1975) & Hipparcos & NSVS \\
& $\mathrm{UB} \mathrm{V}$ & $\mathrm{B} \mathrm{V}$ & $\mathrm{V}$ & $\mathrm{R}$ \\
\hline$i^{o}$ & $80.96 \pm 0.14$ & $81.142 \pm 0.21$ & $86.91 \pm 0.24$ & $85.72 \pm 0.50$ \\
$\mathrm{~T}_{\text {eff }_{1}}(\mathrm{~K})$ & $22400[\mathrm{Fix}]$ & $22400[\mathrm{Fix}]$ & $22400[\mathrm{Fix}]$ & $22400[\mathrm{Fix}]$ \\
$\mathrm{T}_{\text {eff }_{2}}(\mathrm{~K})$ & $10900 \pm 160$ & $11000 \pm 200$ & $10540 \pm 260$ & $11520 \pm 370$ \\
$\Omega_{1}$ & $4.476 \pm 0.090$ & $3.359 \pm 0.049$ & $3.456 \pm 0.024$ & $3.994 \pm 0.150$ \\
$\Omega_{2}$ & $2.332 \pm 0.132$ & $2.332 \pm 0.132$ & $2.332 \pm 0.132$ & $2.333 \pm 0.112$ \\
$r_{1}$ & $0.2374 \pm 0.0057$ & $0.3191 \pm 0.0054$ & $0.3151 \pm 0.0025$ & $0.2678 \pm 0.0109$ \\
$r_{2}$ & $0.2626 \pm 0.0065$ & $0.2625 \pm 0.0044$ & $0.2625 \pm 0.0021$ & $0.2625 \pm 0.0107$ \\
$\frac{L_{1}}{\left(L_{1}+L_{2}\right)} \mathrm{R}$ & - & - & - & $0.7491 \pm 0.0212$ \\
$\frac{L_{1}}{\left(L_{1}+L_{2}\right)} \mathrm{V}$ & $0.7655 \pm 0.0109$ & $0.8766 \pm 0.0082$ & $0.847 \pm 0.006$ & $0.7491 \pm 0.0212$ \\
$\frac{L_{1}}{\left(L_{1}+L_{2}\right)} \mathrm{B}$ & $0.7505 \pm 0.0128$ & $0.8006 \pm 0.0084$ & - & - \\
$\frac{L_{1}}{\left(L_{1}+L_{2}\right)} \mathrm{U}$ & $0.8302 \pm 0.0124$ & - & - & - \\
$\sum(O-C)^{2}$ & 0.848 & 0.987 & 0.987 & 0.988 \\
$N$ & 1446 & 532 & 134 & 107 \\
$\sigma$ & 0.008 & 0.091 & 0.087 & 0.098 \\
\hline
\end{tabular}

system were collected in the framework of two important surveys: $\mathrm{Hp}-$ band data of (Perrvman et al., 1997) and R-band data of the NSVS project (Wozniak et al., 2004). These magnitudes were obtained in a time interval of about three years. The accuracy of the Hipparcos data is about $\sigma_{H_{p}}$ $\sim 0.01$ mag. The $H_{p}$ magnitudes measured by the Hipparcos mission were transformed to the Johnson's V-passband using the transformation coefficients given by Harmaned (1998). All the data obtained by several researchers or surveys are plotted against the orbital phase in Fig. 5.

The most-commonly used code for modelling the light curves of the eclipsing binaries is that of Wilson \& Devinney (1971). This code was up-dated and implemented in the PHOEBE code of Prša \& Zwitter (2005). One of the main difficulties in this modelling is determination of individual effective temperatures of both stars. Generally used practice is to estimate effective tem- 




Figure 5: Comparison of the observed and computed light curves of GT Cep. The continuous lines show the best-fit model. 
perature of primary star and determine that of the secondary star. Effective temperature of the primary star could be estimated from its spectra or color indices. The average apparent visual magnitude and colors are measured by Lacy (1992) as $\mathrm{V}=8.125 \pm 0.017, \mathrm{U}-\mathrm{B}=-0.532 \pm 0.013, \mathrm{~B}-\mathrm{V}=0.339 \pm 0.003$. The quantity $Q=(U-B)-\left(E_{(U-B)} /\left(E_{(B-V)}\right)(B-V)\right.$ is independent of interstellar extinction. The average value obtained is $\left(E_{(U-B)} /\left(E_{(B-V)}\right)=0.72 \pm 0.03\right.$ Johnson \& Morgan (1953); Hovhannessian (2004). We compute the reddeningfree index as $Q=-0.776 \pm 0.013$. The values of index were calculated by Hovhannessian (2004) begining from O8 to G2 spectral type for the luminosity classes between main-sequence and supergiants. A calibration between $Q$ and spectral type yield a spectral type of $\mathrm{B} 1 \mathrm{~V}$, in agreement with spectral classification made from the spectra. In addition the infrared colors $\mathrm{J}-\mathrm{H}=0.096 \pm 0.05, \mathrm{H}-\mathrm{K}=-0.007 \pm 0.05 \mathrm{mag}$ are given in the $2 \mathrm{MASS}$ catalogue (Cutri et al., 2003) correspond to an early B type star.

Logarithmic limb-darkening coefficients were interpolated from the tables of van Hamme (1993). They are updated at every iteration. The gravitybrightening coefficients $g_{1}=g_{2}=1.0$ and albedos $A_{1}=A_{2}=1.0$ were fixed for both components, as appropriate for stars with radiative atmospheres. Since the preliminary analysis indicates that secondary star fills its Roche lobe, synchronous rotations were adopted and Mode 5 was used in the solution. This mode is used for the Algol systems, e.g., secondary star fill their limiting Roche lobes. The BV light curves of Bondarenko \& Tokareva (1975), UBV light curves of Bartolini. Bonifazi. \& Milano (1984), Hipparcos and NSVS were analyzed separately.

The adjustable parameters in the light curves fitting were the orbital 
inclination $i$, the effective temperature of the secondary star $\mathrm{T}_{\text {eff }}$, the luminosity of the primary $\mathrm{L}_{1}$, and the zero-epoch offset. The parameters of our final solution are listed in Table6. The uncertainties assigned to the adjusted parameters are the internal errors provided directly by the code. In the last three lines of Table 6 sums of squares of residuals $\sum(O-C)^{2}$, number of data points $N$, and standard deviations $\sigma$ of the observed light curves are presented, respectively. It is obvious that the $\sigma$-value obtained for

the UBV light curves of Bartolini, Bonifazi, \& Milano (1984) is the smallest, amounting to at least eleventh of the others. Therefore I adopt the parameters obtained from the UBV light curves as the best fit model parameters for GT Cep. The O-C residuals point out a sinusoidal change which has been attributed to a third-body orbit. We repeated the analysis taking the thirdlight as an adjustable parameter. The analysis showed that there is no sign about the existence of the light contribution of an additional component. The computed light curves are compared with the observations in Fig. 5.

\section{RESULTS AND DISCUSSION}

Based on mid-resolution spectroscopic observations I have obtained radial velocities of both components for the high-mass eclipsing binary GT Cep. Analysis of the radial velocities yielded $\mathrm{M}_{1} \sin ^{3} i=10.30 \mathrm{M}_{\odot}, \mathrm{M}_{2} \sin ^{3} i=2.49$ $\mathrm{M}_{\odot}$, and $a \sin i=28.42 \mathrm{R}_{\odot}$. These values are too different from those of the previous determination by Fitzgerald (1964). Combining the results of the simultaneous UBV light curves' analysis, listed in the second column of Table 6, I determined absolute parameters for the components. For calculation of the fundamental stellar parameters for the components such as masses, radii, lu- 
Table 7: Absolute parameters, magnitudes and colours for the components of GT Cep.

\begin{tabular}{lcc}
\hline Parameter & \multicolumn{2}{c}{ GT Cep } \\
& Primary & Secondary \\
\hline Mass $\left(\mathrm{M}_{\odot}\right)$ & $10.70 \pm 0.50$ & $2.58 \pm 0.14$ \\
Radius $\left(\mathrm{R}_{\odot}\right)$ & $6.34 \pm 0.19$ & $6.98 \pm 0.11$ \\
$T_{\text {eff }}(\mathrm{K})$ & $22400 \pm 950$ & $10900 \pm 300$ \\
$\log \left(L / L_{\odot}\right)$ & $4.025 \pm 0.078$ & $2.862 \pm 0.035$ \\
$\log g($ cgs $)$ & $3.798 \pm 0.022$ & $3.094 \pm 0.025$ \\
$S p . T y p e$ & $\mathrm{~B} 2 \mathrm{~V}$ & $\mathrm{~A} 0 \mathrm{IV}$ \\
$M_{\text {bol }}(\mathrm{mag})$ & $-5.31 \pm 0.19$ & $-2.40 \pm 0.09$ \\
$B C(\mathrm{mag})$ & -2.18 & -0.43 \\
$M_{V}(\mathrm{mag})$ & $-3.14 \pm 0.10$ & $-1.97 \pm 0.07$ \\
$(V \sin i)_{\text {calc. }}\left(\mathrm{km} \mathrm{s}^{-1}\right)$ & $70 \pm 2$ & $78 \pm 2$ \\
$(\text { V sin i })_{\text {obs. }}\left(\mathrm{km} \mathrm{s}^{-1}\right)$ & $70 \pm 4$ & $70 \pm 7$ \\
$d$ (pc) & $854 \pm 43$ & $903 \pm 52$ \\
\hline
\end{tabular}

minosities and their formal standard deviations the JKTABSDIM 3 code was used. This code calculates physical parameters and distance to the system using several different sources of bolometric corrections (Southworth et al., 2005). The final results are presented in Table 7.

The luminosities of the components were calculated from their absolute radii and effective temperatures. Using these luminosities I have calculated bolometric absolute magnitudes of the components, assuming solar bolometric absolute magnitude of 4.74 mag. Next I applied a bolometric correction for each star based on our spectral type and the effective temperatures, listed in Table 7, from (Flower, 1996) to obtain their absolute visual magnitudes. Analysis of the light curve yields light ratio of 0.306 for the $\mathrm{V}$-passband. This light ratio, the observed colours and the intrinsic colour of the primary star

\footnotetext{
${ }^{3}$ This can be obtained from http://http://www.astro.keele.ac uk/ jkt/codes.html
} 
of $(B-V)_{0}=-0.25 \pm 0.01$ (Drilling \& Landolt, 2000) allow us to estimate an intrinsic composite colour of $(B-V)_{0}=-0.27 \pm 0.01$. Thus, the interstellar reddening of $E_{(B-V)}=0.61 \pm 0.01 \mathrm{mag}$ and absorption in the $\mathrm{V}$-passband of $A_{V)}=1.89 \pm 0.01 \mathrm{mag}$ are estimated for the system. I arrived at distance modulus for the primary and secondary component as 9.66 and $9.78 \mathrm{mag}$, respectively, by using $V_{1}=8.42$ and $V_{2}=9.70 \mathrm{mag}$. These distance moduli correspond to a distance of $854 \pm 43$ pc for the primary and $903 \pm 52$ pc for the secondary star. Since the secondary star is an evolved star and losing its mass I adopt distance to the system estimated from the primary component.

Figure 6 shows the components, with 1-sigma error bars, of GT Cep in the $\log \mathrm{T}_{\text {eff }}-\log \mathrm{L} / \mathrm{L}_{\odot}$ panel. We used the models of Ekström et al. (2012), for single stars in the range $0.8-120 \mathrm{M}_{\odot}$ at solar metallicity $(\mathrm{Z}=0.014)$, which include rotation to comparison of our measured parameters with evolutionary models. While these evolutionary models are not appropriate for the SDABs, I plot the stars in the well-known Hertzsprung-Russell diagram to show their locations.

In the short-period, $\mathrm{P}<5 \mathrm{~d}$, SDABs the mass-gaining components are large enough, relative to the separation, that the infalling mass can directly impact on the accretor. For the first time, Lubow \& Shu (1975) discussed and modelled formation of discs in semi-detached systems. In Fig.3 of Dervisoglu et al. (2010), the so-called $r-q$ diagram, the SDABs with and without discs are plotted. With a fractional radius of 0.24 and a mass-ratio of 0.24, GT Cep locates in the $r-q$ diagram where the gainers of SDABs without permanent or transient disc are gathered. In Fig.2 of Dervisoglu et al. (2010) the ratios of the observed equatorial to the computed synchronous rotational 


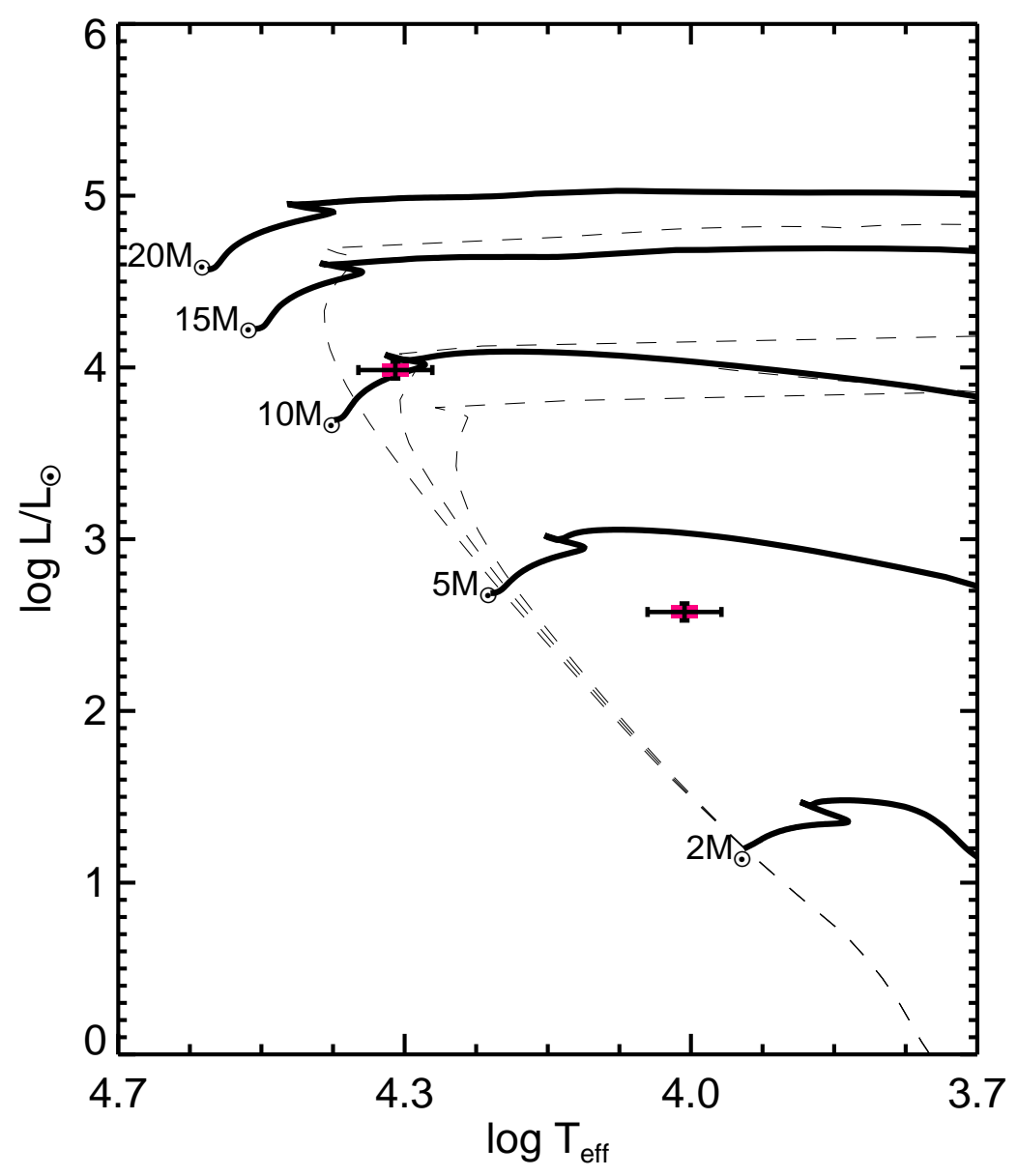

Figure 6: Locations of the components on the effective temperature-luminosity panel. Evolutionary tracks (solid lines) for stars 2, 5, 10, 15 and $20 \mathrm{M}_{\odot}$ and isochrones correspond to 10,20 , and $30 \mathrm{Myr}$ (dashed lines, going from left to right) for single rotating stars with solar metallicity (Ekström et al. 2012). 
velocities for the SDABs were plotted against the mass of the gainers. The mass-gaining primary star of GT Cep is appeared as the highest mass star with synchronous rotational velocities. van Rensbergen et al. (2006) studied evolution of interacting binaries with a B type primary at birth. They have taken into account both loss of mass and angular momentum during binary evolution. Their calculation clearly showed that the binaries have to lose a significant amount of mass without losing much angular momentum. Their liberal scenario with much mass loss without much loss of angular momentum yielded orbital periods and mass ratios of Algols that are in better agreement with the observations. Though physical events related to the liberal scenario at binaries with a B type binary at birth are yet not fully understood they have estimated that only half of the matter lost by the donor is captured by the gainer and the other half left the system. This assumption reveals that a mass of about $3 \mathrm{M}_{\odot}$ is lost for the case of GT Cep during its evolution.

Since we found the period and projected radius of the orbit the mass of the third star can only be computed for different values of orbital inclination. The total mass of the eclipsing pair was obtained as $13.28 \mathrm{M}_{\odot}$. For the inclination of the third-body orbit of $90^{\circ}, 80^{\circ}$ and $70^{\circ}$ I calculate masses for the third star as $6.9,7.0$ and $7.45 \mathrm{M}_{\odot}$, respectively. For a wide range of orbital inclination the mass of third star is about 7 solar masses. If it were a main-sequence star its visual magnitude would be $8.8 \mathrm{mag}$, i.e. 0.4 mag fainter than the primary and 0.9 mag brighter than the secondary component. The third star would be about $0.7 \mathrm{mag}$ fainter from the eclipsing pair. Assuming the distance to the eclipsing pair as $854 \mathrm{pc}$, and separation between the eclipsing pair and the third star as $40 A U$ one can easily calculate an angular separation of about 
0.05 arcseconds. Such a star could be angularly resolved by contemporary instruments and methods. Since no photometric or spectroscopic signature has been observed for the third star up to date it would be reasonable to think that this component either might not be a normal star or it is a multiple star system.

\section{Acknowledgments}

This paper is dedicated to Professor C. Ibanoglu who spent most his life on the structure and evolution of the binary stars. He surveyed, for example, more than thirty years the exotic binary V471 Tauri. He and his colleagues carried out many quantitative studies of the physical properties of the close binaries. I am grateful for his aid in every step of my studies and his valu-

able contributions to close binary systems. We thank to TÜBİTAK National Observatory (TUG) for a partial support in using RTT150 telescope with project number 11BRTT150-198. This study is supported by Turkish Scientific and Technology Council under project number 112T263. We thank to EBILTEM Ege University Research Center for a partial support with project number 2013/BIL/018. The following internet-based resources were used in research for this paper: the NASA Astrophysics Data System; the SIMBAD database operated at CDS, Strasbourg, France; and the ar $\chi$ iv scientific paper preprint service operated by Cornell University.

\section{References}

Bartolini C., Bonifazi A., Milano L., 1984, A\&AS, 55, 403

Berthold, T., 1976, IBVS, 1115, 1B 
Bobylev V. V., Goncharov G. A., Bajkova A. T., 2006, ARep, 50, 733

Bondarenko I. I., Tokareva Y. I., 1975, PZP, 2, 171

Cakirli O., Ibanoglu C. \& Sipahi E., MNRAS, inpress

Cutri R. M., et al., 2003, The IRSA 2MASS All-Sky Point Source Catalog, NASA/IPAC Infrared Science Archive. http://irsa.ipac.caltech.edu/applications/Gator/

Conti, P. S., \& Ebbets, D. 1977, ApJ, 213, 438

De Becker M., Raucq F., 2013, A\&A, 558, A28

Dervisoglu, A., Tout, C. A., \& Ibanoglu, C. 2010, MNRAS, 406, 1071

Diethelm, R., 2003, IBVS, 5438, 1D 01

Drilling J. S., Landolt A. U., 2000, Allen's astrophysical quantities, 4th ed. Edited by Arthur N. Cox. ISBN: 0-387-98746-0. Publisher: New York: AIP Press; Springer, p.381

Ekström, S., Georgy, C., Eggenberger, P., et al. 2012, A\&Ap, 537, A146

ESA, 1997, The Hipparcos \& Tycho Catalogues, SP-1220

Fitzgerald P., 1964, Publication of The David Dunlap Observatory, 2, 415

Flower, P. J., 1996, ApJ, 469, 355

Gray, D. F. 1992, The observation and analysis of stellar photospheres, (2nd ed.) Camb. Astrophys. Ser., Vol. 20 
Harmanec, P. 1998, A\&A, 335, 173

Hovhannessian R. K., 2004, Ap, 47, 499

Ibanoglu, C., Çakirli, Ö., Değirmenci, Ö., et al. 2000, A\&Ap, 354, 188

Ibanoglu, C., Soydugan, F., Soydugan, E., \& Dervisoglu, A. 2006, MNRAS, 373,435

Johnson H. L., Morgan W. W., 1953, ApJ, 117, 313

Johnson D. R. H., Soderblom D. R., 1987, AJ, 93, 864

Kane S. R., Schneider D. P., Ge J., 2007, MNRAS, 377, 1610

Kazarovets E. V., Samus N. N., Durlevich O. V., et al. 1999, IBVS, 4659, 1

Kreiner, M., 2004, AcA, 54, 207K

Lacy, C. H. 1992, AJ, 104, 801

Lacy C. H. S., 2002, AJ, 124, 1162

Lanz, T., \& Hubeny, I. 2007, ApJS, 169, 83

Liao, W.-P., \& Qian, S.-B. 2010, MNRAS, 405, 1930

Lubow, S. H., \& Shu, F. H. 1975, ApJ, 198, 383

Meyer, P., 2006, OEJV, 28, 1M

Mihalas D., Binney J., 1981, in Galactic Astronomy, 2nd edition, Freeman, San Fransisco, p.181 
Penny, L. R. 1996, ApJ, 463, 737

Perryman M. A. C., Lindegren L., Kovalevsky J. et al., 1997, A\&A, 323, L49

Prša A., Zwitter T. 2005, ApJ, 628, 426P

Royer F., Gerbaldi M., Faraggiana R., \& Gomez A. E. 2002, A\&A, 381, 105

Southworth J., Smalley B., Maxted P. F. L., Claret A. \& Etzel P. B. 2005, MNRAS, 363, 529

Simkin S. J., 1974, A\&A, 31, 129

Strohmeier W., Knigge R., Ott H., 1962, Veraffentichungen der RemeisSternwarte Bamberg, vol. 5 no. 13, pp. 3, 10

Strohmeier, W., 1962, IBVS, 9, 1S

Valdes, F., Gupta, R., Rose, J. A., Singh, H. P., \& Bell, D. J. 2004, ApJS, 152,251

van Leeuwen F. 2007, A\&A, 474, 653

van Hamme, W. 1993 AJ, 106, 2096

van Rensbergen, W., De Loore, C., \& Jansen, K. 2006, A\&A, 446, 1071

Wilson R.E. \& Devinney E.J., 1971, ApJ, 166, 605

Wood D. B., 1971, AJ, 76, 701

Wozniak P.R., Vestrand C.W., Akerlof R., et al., 2004, AJ, 127, 2436

Wu Y., Singh H. P., Prugniel P., Gupta R., Koleva M., 2011, A\&A, 525, A71 